\title{
A Clustering Algorithm Based on Power for WSNs*
}

\author{
Kaihua $\mathrm{Xu}^{1}$, Ya Wang ${ }^{1}$, and Yuhua $\mathrm{Liu}^{2}$ \\ ${ }^{1}$ College of Physical Science and Technology, Central China Normal University, Wuhan, \\ 430079, P.R. China, \\ Kaihua Xu, Ya Wang, xihuanni3957@sohu.com \\ ${ }^{2}$ Department of Computer Science, Central China Normal University, Wuhan, 430079, \\ P.R. China, \\ Yuhua Liu, yhliu@mail.ccnu.edu.cn
}

\begin{abstract}
This paper computes the optimum number of cluster heads based on power first, and then proposes the clustering algorithm for WSNs, at last estimates its performances by our emulator. On analysis, clustering time complexity is dependent on the network diameter and it is suitable for small or medium networks. The simulation results show that using this clustering algorithm can generate network topology of optimum number of clusters, the nodes energy is reduced greatly and the lifetime of networks is extended.
\end{abstract}

Keywords: clustering topology, WSN, generation algorithm, cluster heads, node energy.

\section{Introduction}

The main goal of the topology management for WSNs is to form an optimal framework based on data transmission by removing unnecessary communication links between nodes, and the prerequisite is to assure network coverage and connectivity ${ }^{[1]}$. There are two approaches to topology management in WSNs----power control and hierarchical topology organization. Power control mechanisms adjust the power on a per-node basis, so that one-hop neighbor connectivity is balanced and overall network connectivity is ensured; With hierarchical topology control, a subset of the network nodes is selected to serve as the network backbone over which essential network control functions are supported ${ }^{[2]}$. This approach to topology control is often called clustering, and our study on this paper focuses on the hierarchical clustering algorithm for WSNs.

\section{The Clustering Algorithm for WSNs}

\subsection{The Optimum Number of Cluster Heads Based on Power}

To study on the hierarchical clustering algorithm for WSNs, we make the following assumptions:

\footnotetext{
* The work was supported by the Natural Science Foundation of China (Grant No.60673163).
} 
1) The nodes in WSNs are distributed as per a homogeneous spatial Poisson process of intensity $\lambda$ in a 2-dimensional space which is a square area of side $2 a$, and $\lambda=\lambda_{0}+\lambda_{1}$, where the intensity of cluster heads is $\lambda_{1}$, the intensity of non-cluster heads is $\lambda_{0} ; p$ with mean the probability of cluster heads in a WSN, thus $\lambda_{1}=p \lambda$, $\lambda_{0}=(1-p) \lambda$;

2) The total number of nodes in this area is $n$, and the area of this 2-dimensional space is $s, n$ with mean $\lambda s$, where $s=4 a^{2}$;

3) Also assume that the origin of coordinates is at the center of the square, and the base station is located at $(0,2 a)$;

4) We assume a simple model for the radio hardware energy dissipation where the transmitter dissipates energy to run the radio electronics and the power amplifier, and the receiver dissipates energy to run the radio electronics ${ }^{[3]}$.

5) In this algorithm, the cluster heads creates a TDMA schedule telling each noncluster heads when it can transmit message.

According to the assumptions above, We get under the exponent the following expression:

$$
p=\sqrt{\frac{10 \lambda}{0.0061632025 n^{2}}}
$$

The computed values of $p$ and the corresponding values of the number of nodes $n$ and the intensity of nodes $\lambda$ for WSNs are provided in Table 1.

\subsection{Parameters for the Clustering Algorithm}

The weighted clustering algorithm (WCA) is an on-demand distributed clustering algorithm proposed for mobile ad hoc networks ${ }^{[4]}$. It elects a node as a cluster head based on four parameters as follows: the number of neighbors, transmit power, battery-life and mobility rate of the node.

Because the nodes are stationary in WSNs, the considered parameters for the optimizing algorithm of WCA are simple relatively. Consider three parameters as follows: 1) the battery-life of nodes $C_{i_{-} \text {res }}$; 2) The nodes degree-difference $\Delta_{i}=\left|d_{i}-E[N]\right|$, where $E[N]=\lambda_{0} / \lambda_{1}$, and $d_{i}$ means the neighbors' number of nodes $i ; 3)$ The average distance between node and its neighbors $d_{i_{-}}$ave $=D_{i} / d_{i}$, where $D_{i}$ means that the total distance between node $i$ and its neighbors. Consider three parameters above, the combined weight $w_{i}$ for node $i$ in WSNs can be calculated:

$$
w_{i}=w_{1} \cdot \frac{C_{t}}{C_{i_{-} r e s}}+w_{2} \cdot \Delta_{i}+w_{3} \cdot d_{i-a v e}
$$

Where, $C_{t}$ is defined as the initial energy of nodes, and $w_{1}, w_{2}, w_{3}$ are defined respectively as the weight of three parameters, where $w_{1}+w_{2}+w_{3}=1$. When the smaller the weight $w_{i}$, the bigger probability of node $i$ being elected as a cluster head. 
Table 1. The optimum number of cluster heads $p$

\begin{tabular}{|c|c|c|}
\hline Number of nodes $(n)$ & Intensity of nodes $(\lambda)$ & Probability of cluster heads $(p)$ \\
\hline 500 & 1.25 & 0.0901 \\
\hline 1000 & 2.5 & 0.0637 \\
\hline 1500 & 3.75 & 0.0520 \\
\hline 2000 & 5 & 0.0450 \\
\hline 2500 & 6.25 & 0.0403 \\
\hline 3000 & 7.5 & 0.0368 \\
\hline
\end{tabular}

\subsection{The Generation of Clustering}

The generation of clustering procedure consists of two processes: cluster heads election phase and cluster Set-Up phase. During cluster heads election phase, the suitable nodes are elected as cluster heads according to some topology control algorithms for WSNs; during cluster Set-Up phase, the cluster heads broadcast their states using the same transmit energy to the non-cluster heads in the networks, after each node has decided to which cluster it belongs, it must inform the cluster head node that it will be a member of the cluster, and then the framework of WSNs forms.

\section{Simulation Study and Performance Evaluation}

\subsection{Simulation Study}

The most important goal in WSNs is the effective utilization of energy, so in simulation study, we considered the nexus between the number of cluster heads and the total amplifier energy for WSNs. Figure 1 described the function diagram $e_{t}(p)$ for 500, 1000 and 2000 nodes in WSNs, where $e_{t}(p)$ is the total amplifier energy for WSNs. It is evident that the total amplifier energy spent in the network is indeed minimal at the theoretically optimal values of the parameter $p$ computed using (1).

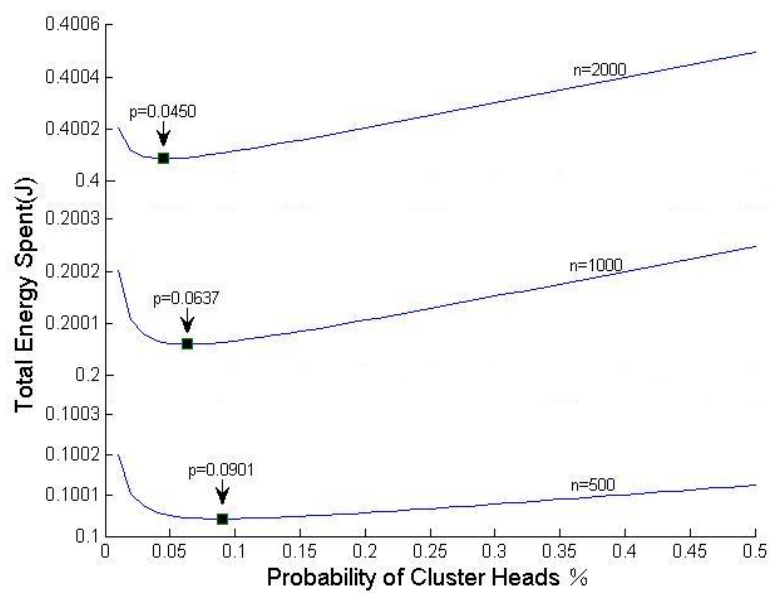

Fig. 1. Total amplifier energy vs. probability of cluster heads in WSNs 


\subsection{Performance Evaluation}

In the clustering algorithm flowchart, its time complexity is $O\left(n^{2}\right)$, where $n$ with mean the number of network nodes. When the size of network is small, the algorithm is simple; when the size of network is large, it is necessary to set more parameters to let it simple. We will study on this research in the future.

\section{Conclusion}

In this paper, take the optimum number of cluster heads based on power and the optimizing algorithm of WCA into consideration, and generate the clustering topology of WSNs. The simulation results show that because of this clustering algorithm, the nodes energy is reduced greatly. This algorithm is suitable for the small and medium networks. When the size of network is large, it is necessary to set more parameters to let it simple. We will study on this research in the future. At same time, what need study on deeply is how to design a routing protocol of security, QoS and so on based on clustering.

\section{References}

1. Akyildiz I F, Su W, Sankarasubramaniam Y, Cayirci E. A survey on sensor networks. IEEE Communications Magazine, 2002, 40: 102 114

2. Bao L, Garcia-Luna-Accves J J. Topology management in ad hoc networks. In: Proc $4^{\text {th }}$ ACM Int'l Symp on mobile Ad Hoc Networking \& Computing (MobiHoc 2003), Annapolis, Maryland. 2003. 129 140

3. S Bandyopadhyay and Edward J.Coyle. An energy-efficient hierarchical clustering algorithm for wireless sensor networks[A].Proc.of IEEE INFOCOM[C].San Francisco, USA: IEEE Computer Society, 2003.1713-1723

4. T. Rappaport, Wireless Communications: Principles \& Practice. Englewood Cliffs, NJ: Prentice-Hall, 1996

5. Mainak Chatterjee, Sajal K Das, Damla Turgut. WCA: A weighted clustering algorithm for mobile ad hoc networks [J]. Journal of Cluster Computing, Special issue on Mobile Ad hoc Networking, 2002, (5): 193 204 